\title{
LAS NORMAS INTERNACIONALES DE INFORMACIÓN FINANCIERA Y LA TOMA DE DECISIONES FINANCIERAS EN LAS EMPRESAS
}

\section{INTERNATIONAL FINANCIAL INFORMATION REGULATIONS AND FINANCIAL DECISIONS IN BUSINESSES}

\author{
Glady Guissela Janampa Gómez \\ Bachiller en Administración de la Universidad San Ignacio de Loyola - Administradora - Portugal Research International \\ Email: guissela.jg@gmail.com (Autor Corresponsal)
}

[Recibido: 10/05/2016 Aceptado: 16/08/2017]

\section{RESUMEN}

El trabajo de investigación tiene por objetivo facilitar la toma de decisiones financieras mediante la aplicación correcta de las normas internacionales de información financiera en las empresas. La investigación ha sido de enfoque cualitativo, de alcance descriptivo y correlacional, diseño no experimental de corte transversal, una población de 135 empresas, la muestra probabilística ha sido de 100 sujetos de estudio, las técnicas utilizadas han sido la encuesta y la recopilación documental, los datos han sido procesados con el programa SPSS. Se llegó a la siguiente conclusión general: La adopción de las Normas Internacionales de Información Financiera (NIIF), incide en gran medida en la toma de decisiones financieras de las empresas; mediante el reconocimiento de las transacciones, la medición de las transacciones, el registro de las transacciones y la presentación razonable de las transacciones empresariales.

\section{PALABRAS CLAVE}

Normas internacionales, información financiera, toma de decisiones.

\begin{abstract}
The purpose of the research work is to facilitate financial decision making through the correct application of international financial reporting standards in companies. The research has been qualitative, descriptive and correlational in scope, non-experimental cross-sectional design, a population of 135 companies, the probabilistic sample has been 100 study subjects, the techniques used have been the survey and the documentary compilation, the data has been processed with the SPSS program. The following general conclusion was reached: The adoption of the International Financial Reporting Standards (IFRS) has a large impact on the financial decision making of companies; through the recognition of transactions, the measurement of transactions, the recording of transactions and the reasonable presentation of business transactions.
\end{abstract}

\section{KEYWORDS}

International standards, financial information, decision-making.

\section{INTRODUCCIÓN}

Las Normas Internacionales de Información Financiera (NIIF) son las normas contables emitidas por el
Consejo de Normas Internacionales de Contabilidad (IASB, por sus siglas en inglés) con el propósito de uniformizar la aplicación de normas contables en el mundo, de manera que sean globalmente aceptadas, comprensibles y de alta calidad. Las NIIF permiten que la información de los estados financieros sea comparable y transparente, lo que ayuda a los inversores y

Como Citar: Janampa, G. (2017). Las normas internacionales de información financiera y la toma de decisiones financieras en las empresas. Quipukamayoc, 25(49), 73-79 . doi: http://dx.doi.org/10.15381/quipu.v25i49.14282 
participantes de los mercados de capitales de todo el mundo a tomar sus decisiones. En el Perú, las NIIF constituyen los Principios de Contabilidad Generalmente aceptados. En la toma de decisiones empresariales importa la elección de un camino a seguir, por lo que en un estado anterior deben evaluarse alternativas de acción. Si estas últimas no están presentes, no existirá decisión. Para tomar una decisión, cualquiera que sea su naturale$\mathrm{za}$, es necesario conocer, comprender, analizar un problema, para así poder darle solución.

Normas internacionales de información financiera

Según Rincón, Lasso y Parrado (2014), las Normas Internacionales de Información Financiera (NIIF) son los principios de contabilidad generalmente aceptados. Son los estándares con los cuales se debe formular la información contable razonable de las empresas. Son las reglas generales para aplicar la metodología contable para el reconocimiento, medición o valoración, registro en libros y presentación de la información en los estados financieros básicos como son el estado de situación financiera, estado de resultado integral, estado de cambios en el patrimonio neto y estado de flujos de efectivo. Estas normas son la garantía de una información contable fiable y de confianza para todos los agentes económicos.

Flores (2015) refiere que las Normas Internacionales de Contabilidad han tomado cada vez mayor aceptación en todo el mundo. Son objetivos del IASB: Desarrollar estándares contables de calidad, comprensibles y de cumplimiento forzoso, que requieran información de alta calidad, trans- parente y comparable dentro de los estados financieros para ayudar a los participantes en los mercados globales de capital y otros usuarios de la información contable en la toma de decisiones económicas; Cooperar activamente con los emisores locales de estándares de contabilidad con el fin de lograr la convergencia definitiva de normas contables en todo el mundo.

Reconocimientos de las transacciones

Ferrer (2015) señala que se reconoce un activo en el balance cuando es probable que se obtengan del mismo beneficio económico futuro para la empresa; además, el activo tiene un costo o valor que puede ser medido con fiabilidad. Se reconoce un pasivo, en el balance, cuando es probable que, del pago de esa obligación presente, se derive la salida de recursos que lleven incorporados beneficios económicos; además, la cuantía del desembolso a realizar pueda ser evaluada con fiabilidad.

\section{Medición de las transacciones}

Ferrer (2015) describe que de acuerdo con las normas contables, la valuación o medición es la determinación de valor de un activo, pasivo y patrimonio. Es decir, es el valor que se va a tener en cuenta para llevarlo a los libros contables. Algunas veces la valuación solo se basa en un documento, otras veces en varios documentos. Esta valuación, algunas veces, se da solo en una fecha; otras veces, se da en varias fechas, según las negociaciones con los clientes, proveedores, acreedores, etc.

Registro de las transacciones

Según lo establecido por Rivera (2015), el Plan Contable General
Empresarial (PCGE) tiene como objetivos: 1) La acumulación de información sobre los hechos económicos que una empresa debe registrar según las actividades que realiza, de acuerdo con una estructura de códigos que cumpla con el modelo contable oficial en el Perú, que es el que corresponde a las Normas Internacionales de Información Financiera - NIIF1. 2) Proporcionar a las empresas los códigos contables para el registro de sus transacciones, que les permitan tener un grado de análisis adecuado; y con base en ello, obtener estados financieros que reflejen su situación financiera, resultados de operaciones y flujos de efectivo; 3) Proporcionar a los organismos supervisores y de control, información estandarizada de las transacciones que las empresas efectúan.

Presentaciones razonables de la información financiera y económica

Pariona (2014) manifiesta que la información financiera y económica razonable se obtiene solamente con la aplicación de las Normas internacionales de información financiera. La información contable razonable está conformada por los estados financieros básicos que contienen los activos, pasivos, ingresos y gastos de la empresa. Dicha información, está formulada con las Normas internacionales de información financiera, es sinónimo de garantía para acceder al financiamiento empresarial.

Anthony (2014) refiere que la información razonable es la información contable digna de fe y confianza. La información posee la cualidad de fidedignidad o fiabilidad cuando está libre de error material y de sesgo o prejuicio, y los usuarios pueden confiar en que es la imagen fiel de lo que pretende representar, o de lo que pue- 
de esperarse razonablemente que represente.

Ferrer (2014) refiere que la información contable es razonable o fiable, si representa fielmente las transacciones financieras, económicas, patrimoniales y demás sucesos que pretende representar, o que se puede esperar razonablemente que represente.

Implementación de las normas internacionales de información financiera: Ayala (2014) manifiesta que la Norma Internacional de Información Financiera 1: La adopción por primera vez de las Normas Internacionales de Información Financiera tiene como objetivo asegurar que los primeros estados financieros, así como sus informes financieros intermedios contengan información de alta calidad, transparente para los usuarios y comparable para todos los periodos en que se presenten. Además, deben suministrar un punto de partida adecuado para la contabilización según las Normas Internacionales de Información Financiera (NIIF); y pueda ser obtenida a un costo que no exceda a sus beneficios.

\section{Estados financieros razonables}

Maldonado (2013) refiere que de acuerdo a la Norma internacional de contabilidad 1: La presentación de estados financieros constituye una representación estructurada de la situación financiera y del rendimiento financiero de una empresa. El objetivo de los estados financieros es suministrar información acerca de la situación financiera: rendimiento financiero y flujos de efectivo de una empresa, que sea útil a una amplia variedad de usuarios a la hora de tomar sus decisiones económicas.

Ferrer (2015) sostiene que los estados financieros deberán presentar razonablemente la situación financiera $y$ el rendimiento financiero, así como los flujos de efectivo de una empresa. Esta presentación razonable requiere la presentación fidedigna de los efectos de las transacciones, así como de otros sucesos y condiciones, de acuerdo con las definiciones y los criterios de reconocimiento de activos, pasivos, ingresos y gastos establecidos.

Toma de decisiones financieras empresariales:

Terry (2013) argumenta que en la toma de decisiones la experiencia es un elemento clave puesto que las decisiones deben tomarse sobre una realidad altamente compleja debido al enorme número de variables que entran en juego. La acumulación de experiencia es larga y costosa. Si consideramos que cuando más se aprende es como consecuencia de los propios errores, el alcanzar un elevado nivel de experiencia en el mundo empresarial puede llegar a tener un coste terriblemente alto.

\section{Decisiones de financiamiento}

Arranz y Acinas (2015) resaltan que las empresas no pueden tener capital de trabajo ni bienes de capital si no toman decisiones de financiamiento para dichos rubros. Financiar las inversiones o activos de las pymes industriales de confecciones es el acto de dotar de dinero y de crédito a dichas empresas, es decir, conseguir recursos y medios de pago para destinarlos a la adquisición de bienes y servicios, necesarios para el desarrollo de las correspondientes actividades económicas. Existen varias fuentes de financiación en las empresas y sobre alguna o algunas deben tomarse decisiones en determinado momento.

\section{Decisiones de inversión}

Weston (2014) sostiene que las empresas necesitan llevar a cabo decisiones de inversión para poder disponer del capital de trabajo o activo corriente, y de los bienes de capital o activos no corrientes. Sin estas inversiones es imposible desarrollar el giro o actividad de estas empresas. Al respecto, decidir es llevar a cabo el proceso por el cual se establecen, analizan y evalúan alternativas.

\section{Decisiones de rentabilidad}

Flores (2015) refiere que la rentabilidad de las empresas es la ganancia que una persona recibe por poner sus ahorros en una institución financiera y se expresa a través de los intereses. La rentabilidad es la ganancia que una persona recibe por poner sus ahorros en una institución financiera y se expresa a través de los intereses, que corresponden a un porcentaje del monto de dinero ahorrado.

\section{Decisiones sobre riesgos}

Mascareñas (2013) sostiene que las empresas deben tomar decisiones sobre riesgos sobre sus deudas, inversiones y la rentabilidad de sus actividades. Al respecto, el riesgo es la probabilidad de un evento adverso y sus consecuencias. El riesgo financiero se refiere a la probabilidad de ocurrencia de un evento que tenga consecuencias financieras negativas para una organización.

Decisiones de liquidez, gestión y solvencia empresarial

Mascareñas (2013) resalta que la liquidez, gestión y solvencia son tres conceptos básicos en las finanzas de una empresa, tres conceptos que encierran un significado diferente y que 
la empresa debe tener unas nociones básicas sobre las características que diferencia cada uno de estos conceptos. No solo porque cada uno encierra conceptos diferentes, sino porque comprenderlos adecuadamente hará tomar buenas o malas decisiones que sin duda repercutirán en el negocio de una u otra manera.

\section{Economía empresarial}

Koontz y O'Donnell (2014) manifiestan que la economía empresarial en el uso de los recursos está relacionada con los términos y condiciones bajo los cuales las empresas adquieren recursos, sean estos financieros, humanos, físicos o tecnológicos (computarizados), se obtiene la cantidad requerida al nivel razonable de calidad en la oportunidad y lugar apropiado, al menor costo posible. Si nos centramos en la economía será importante definir correctamente los gastos.

\section{Eficiencia empresarial}

Koontz y O'Donnell (2014) señalan que la eficiencia empresarial es el resultado positivo luego de la racionalización adecuada de los recursos, acorde con la finalidad buscada por los responsables de la gestión empresarial de las empresas. La eficiencia empresarial está referida a la relación existente entre los bienes o servicios

Tabla 1 .

¿Las Normas internacionales de información financiera son estándares para reconocer, medir, registrar y presentar la información financiera y económica? Fuente: Encuesta realizada

\begin{tabular}{cccc}
$\mathbf{N}^{\circ}$ & Alternativas & Cantidad & $\%$ \\
1 & Totalmente en desacuerdo & 14 & 14,00 \\
\hline 2 & En desacuerdo & 00 & 0,00 \\
\hline 3 & Neutral & 00 & 00,00 \\
\hline 4 & De acuerdo & 00 & 00,00 \\
\hline 5 & Totalmente de acuerdo & 86 & 86,00 \\
\hline & Total & $\mathbf{1 0 0}$ & $\mathbf{1 0 0 , 0 0}$ \\
\hline
\end{tabular}

producidos o entregados y los recursos utilizados para ese fin (productividad), en comparación con un estándar de desempeño establecido.

\section{Efectividad empresarial}

Koontz y O'Donnell (2014) sostienen que la efectividad empresarial se refiere al grado en el cual las empresas logran las metas, objetivos, misión u otros beneficios que pretende alcanzar, previstos en la legislación, fijados por la gerencia y/o exigidos por los clientes. Si nos centramos en la efectividad, debiéramos comenzar por identificar las metas de los programar, por consecuencia operacionalizar las metas con el objetivo de medir la efectividad.

\section{Competitividad empresarial}

Redondo (2015) refiere que la competitividad empresarial es la capacidad de las empresas para desarrollar, mantener unas ventajas comparativas que le permiten disfrutar y sostener una posición destacada en el entorno socio económico en que actúa. Se entiende por ventaja comparativa aquella habilidad, recurso, conocimiento, atributos, etc., que dispone una empresa, de la que carecen sus competidores y que hace posible la obtención de unos rendimientos superiores a estos".

\section{Mejora continua empresarial}

Urteaga (2013) señala que la mejora continua empresarial está referida a la innovación que se realicen en las empresas. Se entiende como tal a los cambios en los rubros, tipos de confecciones, actividades $y$ funciones de las industrias de confecciones; así como en los procesos con el fin de reducir costos, gastos, tiempos, movimientos y otros aspectos relacionados. También se entiende como mejora continua al proceso de gestión relacionado al cumplimiento de las acciones, políticas, metas, objetivos, misión y visión de la empresa en el marco de la permanente innovación que se lleva a cabo.

\section{MATERIAL Y MÉTODOS}

En esta investigación se ha utilizado los siguientes métodos:

Descriptivo.- Para describir todos los aspectos relacionados con las Normas internacionales de información financiera y la toma de decisiones financieras en las Empresas.

Inductivo.- Para inferir las Normas internacionales de información financiera en la toma de decisiones financieras en las empresas. También se inferirá los resultados de la muestra en la población.

Deductivo. Para sacar las conclusiones de las Normas internacionales de información financiera y la toma de decisiones financieras en las Empresas.

\section{RESULTADOS}

Los resultados del estudio de esta investigación se basa en una población de 135 empresas del sector construcción y la muestra probabilística es de 100 sujetos del área contable, de las carreras de administración y contabilidad. 
Este resultado favorece el modelo de investigación desarrollado; por cuanto el $86 \%$ de los encuestados acepta que las Normas internacionales de información financiera son estándares para reconocer, medir, registrar y presentar la información financiera y económica de las Empresas.

Los activos, pasivos, ingresos y gastos de las empresas se reconocen por los ingresos que van a generar en el futuro y por su cuantificación correspondiente. Mientas que el registro de los mismos elementos antes indicados se lleva a cabo mediante un contrato, comprobante de pago, título valor, o si fuera el caso un informe pericial cuando no se disponga de documentación correspondiente. Por su parte el registro de los activos, pasivos, ingresos y gastos se lleva a cabo en base al Plan Contable General para Empresas, el mismo que está armonizado con las Normas Internacionales de Información financiera. Luego la presentación de la información financiera se realiza en el Estado de Situación Financiera (ESF), Estado de Cambios en el Patrimonio Neto (ECPN) y Estado de Flujos de Efectivo (EFE); mientras que la situación económica se presenta en el Estado de Resultados Integrales (ERI); dicha presentación se completa con las Notas a los estados financieros que detallan la información de los diferentes rubros de los activos y pasivos en la situación financiera y de los ingresos y gastos de la situación económica.

La información financiera y económica obtenida en base a las Normas Internacionales de Información Financiera, para que tenga razonabilidad, debe ser auditada y sobre dicho examen de auditoría se realiza el análisis estructural para comprender la estructura de los activos, pasivos, ingresos y gastos; luego para saber cómo han evolucionado se aplica el análisis de tendencias; luego se complementa con el análisis con ratios financieros, de liquidez, gestión, solvencia y rentabilidad. Sobre la base de la información analizada se toman las decisiones sobre activos, pasivo, ingresos y gastos; sobre los recursos humanos, Tabla 2.

Encuesta: ¿La toma de decisiones financieras es una toma de postura en relación con las inversiones, financiamiento, rentabilidad, riesgos y otros aspectos? Fuente: Encuesta realizada

\begin{tabular}{|c|c|c|c|c|}
\hline $\mathbf{N}^{\circ}$ & Alternativas & & Cantidad & $\%$ \\
\hline 1 & Totalmente en desacuerdo & & 15 & 15.00 \\
\hline 2 & En desacuerdo & & 00 & 0,00 \\
\hline 3 & Neutral & & 00 & 0,00 \\
\hline 4 & De acuerdo & & 00 & 00,00 \\
\hline \multirow[t]{2}{*}{5} & Totalmente de acuerdo & & 85 & 85,00 \\
\hline & & Total & 100 & 100,00 \\
\hline
\end{tabular}

Este resultado favorece el modelo de investigación desarrollado; por cuanto el $85 \%$ de los encuestados acepta que la toma de decisiones financieras es una toma de postura en relación con las inversiones, financiamiento, rentabilidad, riesgos y otros aspectos de las empresas.

A continuación se presenta la hipótesis de investigación, que debidamente contrastada permitirá determinar el grado y tipo de asociatividad entre la variable independiente que es la NIFF y la toma de decisiones en la empresa. Que es la dependiente.

Plateada la hipótesis también conllevara a ser validada mediante la prue-

Tabla 3. materiales, financieros y tecnológicos; sobre las actividades de comercio, industria y servicios y sobre los procesos tales como planeación, organización dirección y control empresarial. Solo la información financiera y económica razonable asegura una buena toma de decisiones empresariales.

\section{Correlación entre las variables / Fuente: Encuesta realizada}

\begin{tabular}{cccc} 
Variables de la & Indicadores estadísticos & $\begin{array}{c}\text { Normas internacio- } \\
\text { nales de información } \\
\text { financiera }\end{array}$ & $\begin{array}{c}\text { Toma de decisiones } \\
\text { financieras } \\
\text { empresariales }\end{array}$ \\
$\begin{array}{c}\text { Normas interna- } \\
\text { cionales de infor- } \\
\text { mación financiera }\end{array}$ & Correlación de Spearman & $100 \%$ & $0,864 \%$ \\
\cline { 2 - 4 } & Sig. (bilateral) & 100 & $10043 \%$ \\
\cline { 2 - 4 } $\begin{array}{c}\text { Toma de decisio- } \\
\text { nes financieras } \\
\text { empresariales }\end{array}$ & Correlación de Spearman & $0,864 \%$ & $100 \%$ \\
\cline { 2 - 4 } & Sig. (bilateral) & $0,043 \%$ & 100 \\
\cline { 2 - 4 } & Muestra & 100 &
\end{tabular}

ba estadística de Spearman, lo cual le dará mayor consistencia a la relación de dependencia de las variables analizadas y con ello se robustecerá el trabajo de investigación

\section{Hipótesis}

H0: Las normas Internacionales de Información financiera (NIIF) no se relacionan con la Toma de Decisiones Financieras Empresariales (TDFE)

$\mathrm{H} 1$ : Las normas Internacionales de Información financiera (NIIF) se relacionan con la Toma de Decisiones Financieras Empresariales (TDFE) Los resultados de la contrastación de la Hipótesis con Sistema SPSS, son los siguientes: 
Esta tabla mide el grado de relación entre las variables independiente $y$ dependiente. Dentro de ello el coeficiente de correlación y el grado de significancia.

En la presente investigación el valor de la correlación es igual a $86.40 \%$, lo cual indica correlación directa, alta y por tanto apoya el modelo de investigación desarrollada.

En base a la Tabla del SPSS tenemos un valor de significancia (Sig), igual a $4,30 \%$, el mismo que es menor al margen de error propuesto del $5.00 \%$ en la investigación, lo que, de acuerdo con la teoría estadística generalmente aceptada, permite rechazar la hipótesis nula y aceptar la hipótesis alternativa, desde el punto de vista de la correlación de las variables. Luego, esto significa que la correlación obtenida para la muestra es significativa y que dicho valor no se debe a la casualidad, sino a la lógica y sentido del modelo de investigación formulado.

\section{DISCUSIÓN}

El valor de la correlación es igual a $86,40 \%$, lo cual indica una interacción o enlace directo entre las variables de nivel alto, lo que apoya el modelo de investigación desarrollado.

El valor de significancia (Sig), igual a 0,043 en la correlación de las variables, el mismo que es menor al margen de error propuesto del 0.05 propuesto en la investigación, lo que de acuerdo con la teoría estadística generalmente aceptada permite rechazar la hipótesis nula y aceptar la hipótesis alternativa, desde el punto de vista de la correlación de las variables.

En base a la correlación de Pearson y el grado de significancia (sig) se determina que la correlación obtenida para la muestra es significativa y que dicho valor no se debe a la casualidad, sino a la lógica y sentido del modelo de investigación desarrollado.

En base a los resultados se determina que la adopción de las Normas Internacionales de Información Financiera (NIIF) incide en grado estadísticamente significativo en la toma de decisiones financieras de las Empresas mediante el reconocimiento de las transacciones, la medición de las transacciones, el registro de las transacciones y la presentación razonable de las transacciones empresariales. 


\section{REFERENCIAS BIBLIOGRÁFICAS}

Anthony, R. (2014). La contabilidad en la administración de empresas. Ciudad de México: Unión Tipográfica Editorial Hispano Americana.

Arranz, A. \& Acinas, J. (2015). Rentabilidad y Mejora Continua. Madrid: Editorial Donostiarra S.A.

Ayala, P. (2014). Estados financieros con NIIF. Lima: Actualidad Empresarial.

Ferrer, A. (2014). Formulación, Análisis e interpretación de Estados Financieros. Lima: Pacífico Editores.

Ferrer, A. (2015). Estados financieros con NIIF. Lima: Editorial Ferrer Quea.

Ferrer, A. (2015). Normas internacionales de información financiera. Lima: Editorial Ferrer Quea.
Flores, L. (2015). Normas internacionales de información financiera. Lima: Actualidad empresarial.

Flores. J. (2015). Gestión Financiera. Lima: CECOF Asesores.

Koontz, H. \& O’Donnell, C. (2014). Administración Moderna. Ciudad de México: Litográfica Ingramex S.A.

Maldonado, R. (2013). Contabilidad con NIIF. Lima: Editorial San Marcos

Mascareñas, J. (2013). Rentabilidad $y$ riesgo financiero. Madrid: Editorial Días de Santos.

Pariona, L. (2014). Información contable para la toma de decisiones empresariales. Lima: Pacífico Editores.

Redondo, A. (2015). Finanzas empresariales. Caracas: Editorial Centro Contable Venezolano.
Rincón, C., Lasso G. y Parrado, A. (2014). Contabilidad Siglo XXI. Bogotá: ECOE Ediciones.

Rivera, L. (2015). Contabilidad empresarial. Lima: Editorial Los Andes.

Romero, A. (2015). Información financiera empresarial. Lima: Editorial San Marcos.

Terry, G. (2013). Principios de Administración. Ciudad de México: Prentice-Hall Hispanoamericana SA.

Urteaga, R. (2013). Mejora continua empresarial. Ciudad de México: Litográfica Ingramex S.A.

Weston, J. (2014). Finanzas. Madrid: Editorial Donostiarra S.A. 